\title{
VARIETIES CHARACTERIZED BY THEIR ENDOMORPHISMS
}

\author{
Rafael B. Andrist and Hanspeter Kraft
}

\begin{abstract}
We show that two varieties $X$ and $Y$ with isomorphic endomorphism semigroups are isomorphic up to field automorphism if one of them is affine and contains a copy of the affine line. A holomorphic version of this result is due to the first author.
\end{abstract}

\section{Introduction}

It is a well-known fact that an affine variety $X$ over an algebraically closed field $k$ is determined (up to isomorphism) by its $k$-algebra of polynomial functions $\mathcal{O}(X)$. It is natural to ask whether other algebraic structures like the group of automorphisms or the semigroup of endomorphisms could determine a variety.

In general, the automorphism group might consist only of the identity, and the endomorphism semigroup might consist of the identity and the constant self-maps (see Proposition 3.1). Considering only automorphisms is usually hopeless, and the situation is not even clear in the case of $\mathbb{C}^{n}$ where the automorphism group is huge. The advantage of semigroups lies in the natural one-to-one correspondence between points of the variety and its constant maps. Moreover, any such isomorphism of semigroups $\Phi: \operatorname{End}(X) \rightarrow \operatorname{End}(Y)$ is induced by conjugation with a map $\varphi: X \rightarrow Y$, i.e., $\Phi(f)=\varphi \circ f \circ \varphi^{-1}$ (see Remark 2.3).

For topological spaces and continuous endomorphisms, the characterization by endomorphisms has been studied in detail, see e.g., the survey by Magill [Mag75].

For complex manifolds and holomorphic endomorphisms, the question has been investigated first by Hinkkannen in 1992 [Hin92] who showed with elementary methods that a map $\varphi: \mathbb{C} \rightarrow \mathbb{C}$ which conjugates endomorphisms of $\mathbb{C}$ (i.e., entire holomorphic functions on $\mathbb{C}$ ) to endomorphisms of $\mathbb{C}$ is a composition of a continuous field isomorphism and a holomorphic automorphism of $\mathbb{C}$, i.e., $\varphi(z)=a z+b$ or $\varphi(z)=a \bar{z}+b$ with $a, b \in \mathbb{C}, a \neq 0$.

In 1993, Eremenko [Ere93] proved for Riemann surfaces admitting non-constant bounded holomorphic functions that they are determined by their semigroup of endomorphisms. His result and method of proof was extended to bounded domains in $\mathbb{C}^{n}$ by Merenkov [Mer02] in 2002 and to the case of $\mathbb{C}^{n}$ by Buzzard and Merenkov [BM03] in 2003.

The case of $\mathbb{C}^{n}$ was generalized to Stein manifolds which contain a properly embedded copy of the affine complex line by the first author [And11] using a different method. The basic idea is to consider $\mathcal{O}(X)$ as a subset of $\operatorname{End}(X)$ with the help of the embedded affine line, and the crucial part is to identify the affine line after conjugation. This is possible due to the generalization of Hartogs' theorem on separate

Received by the editors January 27, 2013. 
analyticity [Har06] to complex Lie groups and making use of the fact that the affine line can be viewed as a Lie group.

We will solve here the problem in the algebraic setting, see Theorem 2.1 below.

Remark 1.1. If an affine variety $X$ admits a unipotent automorphism $u$ of infinite order, then it contains many affine lines, namely the non-trivial orbits under the action of the additive group $k^{+}:=\overline{\langle u\rangle}$. The existence of such actions is measured by the so-called Makar-Limanov-invariant; see [ML96].

\section{The main results}

From now on we assume that the base field $k$ is algebraically closed of arbitrary characteristic. We will confuse the affine line $\mathbb{A}^{1}$ with the field $k$ so that $\mathbb{A}^{1}$ has the structure of a field. In our setting, a variety $X$ is a $k$-variety, and $X$ is not necessarily irreducible.

Theorem 2.1. Let $X$ and $Y$ be varieties and assume that there exists an isomorphism $\operatorname{End}(X) \simeq \operatorname{End}(Y)$ of semigroups. If $X$ is affine and contains a closed subvariety isomorphic to $\mathbb{A}^{1}$, then $X \simeq Y_{\sigma}$ where $\sigma$ is an automorphism of the base field $k$.

(Here $Y_{\sigma}$ denotes the variety obtained from $Y$ by twisting with the morphism $\operatorname{Spec} \sigma^{-1}:$ Spec $k \rightarrow \operatorname{Spec} k$.)

There is a canonical embedding $X \hookrightarrow \operatorname{End}(X)$ by sending $x \in X$ to the constant map $\gamma_{x}$ with value $x$. The following lemma is clear.

Lemma 2.2. The endomorphism $\gamma \in \operatorname{End}(X)$ is a constant map if and only if $\gamma \circ f=$ $\gamma$ for all $f \in \operatorname{End}(X)$. Moreover, we have $f \circ \gamma_{x}=\gamma_{f(x)}$ for any $f \in \operatorname{End}(X)$ and $x \in X$.

Remark 2.3. The lemma implies that an isomorphism $\Phi: \operatorname{End}(X) \stackrel{\sim}{\longrightarrow} \operatorname{End}(Y)$ induces a bijection $\varphi: X \rightarrow Y$, because $\Phi\left(\gamma_{x}\right)$ is again a constant function, hence of the form $\gamma_{y}$ for some $y \in Y$. Moreover, we get $\Phi(f)=\varphi \circ f \circ \varphi^{-1}$ for all $f \in \operatorname{End}(X)$. In fact, this is clear for the constant maps, by definition of $\varphi$, and then follows for all $f \in \operatorname{End}(X)$ from the second part of the lemma.

This leads to the following definition.

Definition 2.4. A map $\varphi: X \rightarrow Y$ is called conjugating if it is bijective and induces a homomorphism $\Phi: \operatorname{End}(X) \rightarrow \operatorname{End}(Y)$ by $f \mapsto \varphi \circ f \circ \varphi^{-1}$. It is called iso-conjugating if, in addition, the induced homomorphism $\Phi: \operatorname{End}(X) \rightarrow \operatorname{End}(Y)$ is an isomorphism.

Now the theorem above is a consequence of the next result.

Theorem 2.5. Let $X, Y$ be two k-varieties and $\varphi: X \rightarrow Y$ an iso-conjugating map. If $X$ is affine and contains a closed subvariety isomorphic to $\mathbb{A}^{1}$, then there is an automorphism $\sigma$ of $k$ such that the composition of $\varphi$ with the canonical map $Y \rightarrow Y_{\sigma}$ is an isomorphism $X \stackrel{\sim}{\longrightarrow} Y_{\sigma}$ of varieties. 


\section{Endo-free varieties}

The following shows that the theorems do not hold if we drop the assumption that one of the varieties contains a copy of the affine line. Call a variety $X$ endo-free if the only endomorphisms are the identity and the constant maps. In this case we can identify $\operatorname{End}(X)$ with the semigroup $\{$ id $\} \cup X$ where the multiplication on $X$ is given by $x \circ y=x$. This shows that every bijective map $\varphi: X \rightarrow Y$ between two endo-free varieties is iso-conjugating. Hence Theorem 2 cannot hold for an endo-free variety.

Proposition 3.1. If $k$ is not the algebraic closure of a finite field, then there exist endo-free smooth affine $k$-varieties of arbitrary dimension.

As pointed out by the referee the assumption on $k$ is necessary. In fact, for $k=\overline{\mathbb{F}_{p}}$ any $k$-variety is defined over a finite field and thus admits the Frobenius endomorphism.

Let $A$ be a simple abelian variety with a polarization $\lambda_{\mathcal{L}}: A \rightarrow A^{\vee}$ defined by a very ample invertible sheaf $\mathcal{L}$. Recall that $\lambda_{\mathcal{L}}(a):=t_{a}^{*} \mathcal{L} \otimes \mathcal{L}^{-1}$ where $t_{a}: A \rightarrow A$ is the translation $x \mapsto x+a$. Denote by $F:=\operatorname{ker} \lambda_{\mathcal{L}}$ the finite kernel of $\lambda_{\mathcal{L}}$. It follows from Bertini's Theorem (see [Har77, Chapter II, Theorem 8.18]) that there is a prime divisor $D$ on $A$ such that $\mathcal{L}=\mathcal{L}(D)$ and that $\operatorname{Supp}(D) \cap F=\{0\}$ where $\operatorname{Supp}(D) \subset A$ denotes the underlying hypersurface. Moreover, $A \backslash \operatorname{Supp}(D)$ is affine. For $a \in A$ we use the notation $D_{a}$ to denote the translated divisor $t_{a}(D)=D+a$, so that $\mathcal{L}\left(D_{a}\right)=t_{-a}^{*} \mathcal{L}(D)$.

Lemma 3.2. If $\operatorname{Supp}\left(D_{a}\right)=\operatorname{Supp}\left(D_{b}\right)$, then $a=b$.

Proof. If $\operatorname{Supp}\left(D_{a}\right)=\operatorname{Supp}\left(D_{b}\right)$, then $D_{a}=D_{b}$ as divisors and so $\lambda_{\mathcal{L}}(-a)=\lambda_{\mathcal{L}}(-b)$, hence $a-b \in F=\operatorname{ker} \lambda_{\mathcal{L}}$. Since $0 \in \operatorname{Supp}(D)$ we get $a \in \operatorname{Supp}\left(D_{a}\right)=\operatorname{Supp}(D)+a=$ $\operatorname{Supp}(D)+b$, hence $a-b \in \operatorname{Supp}(D)$ which implies $a=b$.

Lemma 3.3. Let $\varphi: A \rightarrow A$ be a morphism, and let $a \in A$ be an element of infinite order. If $\varphi(\operatorname{Supp}(D)) \subseteq \operatorname{Supp}(D)$ and $\varphi\left(\operatorname{Supp}\left(D_{a}\right)\right) \subseteq \operatorname{Supp}\left(D_{a}\right)$, then $\varphi=\mathrm{id}$.

Proof. Every morphism $\varphi: A \rightarrow A$ is the composition of a homomorphism with a translation: $\varphi(x)=\rho(x)+d$ ([Mil08, Corollary 1.2]). Since $A$ is simple and the image of $\varphi$ is not a single point, it follows that $\varphi$ and $\rho$ are both surjective, hence $\varphi(\operatorname{Supp}(D))=\operatorname{Supp}(D)=\rho(\operatorname{Supp}(D))+d$. It follows that $\operatorname{Supp}(D)+a=\operatorname{Supp}\left(D_{a}\right)=$ $\varphi\left(\operatorname{Supp}\left(D_{a}\right)\right)=\varphi(\operatorname{Supp}(D))+\rho(a)=\operatorname{Supp}(D)+\rho(a)$, and so $\rho(a)=a$ by Lemma 3.2. This implies that $\rho(n a)=n a$ for all $n \in \mathbb{Z}$, hence $\rho=$ id, because $\mathbb{Z} a \subset A$ is Zariski-dense. Finally, we have $\operatorname{Supp}(D)=\varphi(\operatorname{Supp}(D))=\operatorname{Supp}(D)+d$, and so $d=0$, hence $\varphi=\mathrm{id}$.

Lemma 3.4. Let $a_{1}, \ldots, a_{m} \in A$ be distinct elements of infinite order where $m \geq 1$. Then every non-constant morphism $\psi: A \backslash \operatorname{Supp}\left(D+D_{a_{1}}+\cdots+D_{a_{m}}\right) \rightarrow A \backslash \operatorname{Supp}(D+$ $\left.D_{a_{1}}+\cdots+D_{a_{m}}\right)$ is an isomorphism of finite order which extends to $A$.

Proof. The morphism $\psi$ extends to a (finite surjective) morphism $\varphi: A \rightarrow A$, because every rational map from a non-singular variety to an abelian variety is regular, see [Mil08, Theorem 3.2]. By construction, $\varphi\left(\operatorname{Supp}\left(D+D_{a_{1}}+\cdots+D_{a_{m}}\right)\right)=\operatorname{Supp}(D+$ $\left.D_{a_{1}}+\cdots+D_{a_{m}}\right)$, hence $\varphi$ permutes the irreducible components $\operatorname{Supp}\left(D_{a_{i}}\right)$. Therefore, a suitable power of $\varphi$ leaves every $\operatorname{Supp}\left(D_{a_{i}}\right)$ invariant, hence satisfies the assumptions of Lemma 3.3, and the claim follows. 
Recall that the isomorphisms of $A$ stabilizing $D$ form a finite group $\operatorname{Iso}_{D}(A)$, because $D$ is very ample. In fact, the linear system $|D|$ defines a closed embedding of $\operatorname{Iso}_{D}(A)$ into some $\mathrm{PGL}_{n}(k)$, hence $\operatorname{Iso}_{D}(A)$ is a linear algebraic group, and every linear algebraic group of isomorphisms of an abelian variety is finite.

Proposition 3.5. Let $a, b \in A$ be two elements of infinite order and assume that

(1) $b \neq \pm a, a \neq 2 b, b \neq 2 a$;

(2) For any automorphism $\rho \in \operatorname{Aut}(A)$ of order 3 of the form $\rho=\psi-\psi(0)$ where $\psi \in \operatorname{Iso}_{D}(A)$, we have $b \neq a+\rho(a)$ and $a \neq b+\rho(b)$.

Then the variety $A \backslash \operatorname{Supp}\left(D+D_{a}+D_{b}\right)$ is endo-free.

Proof. Any isomorphism $\varphi: A \stackrel{\sim}{\longrightarrow} A$ of order 2 is a either a translation $t_{c}$ with a 2torsion element $c \in A_{2}$, or it is of the form $\varphi(x)=-x+d$. Similarly, an isomorphism $\varphi: A \stackrel{\sim}{\longrightarrow} A$ of order 3 is either a translation $t_{c}$ with a 3 -torsion element $c \in A_{3}$, or it is of the form $\varphi(x)=\rho(x)+d$ where $\rho$ is an automorphism of order 3 and $d+\rho(d)+\rho^{2}(d)=0$.

Now let $\psi: A \backslash \operatorname{Supp}\left(D+D_{a}+D_{b}\right) \rightarrow A \backslash \operatorname{Supp}\left(D+D_{a}+D_{b}\right)$ be a non-constant morphism. By Lemma 3.4, $\psi$ extends to an isomorphism $\varphi: A \stackrel{\sim}{\longrightarrow} A$ permuting the hypersurfaces $\operatorname{Supp}(D), \operatorname{Supp}\left(D_{a}\right), \operatorname{Supp}\left(D_{b}\right)$. If $\varphi$ is not the identity, then we are in one of the following three cases.

(i) $\varphi$ has order 2 and fixes $\operatorname{Supp}(D)$. Then $\varphi(x)=-x+d$ and so $\operatorname{Supp}(D)=$ $\varphi(\operatorname{Supp}(D))=-\operatorname{Supp}(D)+d$. Hence, $\varphi\left(\operatorname{Supp}\left(D_{a}\right)\right)=-(\operatorname{Supp}(D)+a)+d=$ $\operatorname{Supp}(D)-a$ which is different from $\operatorname{Supp}(D)+b$ by assumption. So this case cannot occur.

(ii) $\varphi$ has order 2 and fixes $\operatorname{Supp}\left(D_{b}\right)$. Again $\varphi(x)=-x+d$, and $\operatorname{Supp}\left(D_{a}\right)=$ $\operatorname{Supp}(D)+a=\varphi(\operatorname{Supp}(D))=-\operatorname{Supp}(D)+d$. But then

$$
\varphi\left(\operatorname{Supp}\left(D_{b}\right)\right)=-(\operatorname{Supp}(D)+b)+d=\operatorname{Supp}(D)+a-b
$$

which is different from $\operatorname{Supp}\left(D_{b}\right)=\operatorname{Supp}(D)+b$, by assumption. So this case cannot occur either, as well as the case where $\varphi$ fixes $\operatorname{Supp}\left(D_{a}\right)$.

(iii) $\varphi$ has order 3. We can clearly assume that $\varphi(\operatorname{Supp}(D))=\operatorname{Supp}\left(D_{a}\right)$ and that $\varphi\left(\operatorname{Supp}\left(D_{a}\right)\right)=\operatorname{Supp}\left(D_{b}\right)$. Moreover, $\varphi(x)=\rho(x)+d$ with an automorphism $\rho$ of order 3. In addition, $\psi:=\varphi-a \in \operatorname{Iso}_{D}(A)$ and $\rho=\psi-\psi(0)$. First we get $\operatorname{Supp}\left(D_{a}\right)=\operatorname{Supp}(D)+a=\varphi(\operatorname{Supp}(D))=\rho(\operatorname{Supp}(D))+d$, and then

$$
\begin{aligned}
\operatorname{Supp}(D)+b & =\operatorname{Supp}\left(D_{b}\right)=\varphi\left(\operatorname{Supp}\left(D_{a}\right)\right)=\varphi(\operatorname{Supp}(D)+a)= \\
& =\rho(\operatorname{Supp}(D))+\rho(a)+d=\operatorname{Supp}(D)+a+\rho(a),
\end{aligned}
$$

hence $b=a+\rho(a)$, contradicting assumption (2).

Thus $\varphi=$ id and the claim follows.

Proof of Proposition 3.1. Let $A$ be a simple abelian variety. If $k$ is not the algebraic closure of a finite field, then the elements of $A$ of infinite order form a dense set. Moreover, the conditions (1) and (2) of Proposition 3.5 define a non-empty open set of $A \times A$, because $\operatorname{Iso}_{D}(A)$ is finite. Thus we can find pairs $(a, b)$ of elements of $A$ of infinite order satisfying the assumptions of Proposition 3.5. 


\section{Iso-conjugating maps}

For $S \subset \operatorname{End}(X)$ and $x_{0} \in X$ we define the following zero set:

$$
\mathcal{V}\left(S, x_{0}\right):=\left\{x \in X \mid f(x)=x_{0} \text { for all } f \in S\right\} \subset X .
$$

This is clearly a closed subset of $X$.

Lemma 4.1. If $\varphi: X \rightarrow Y$ is a conjugating map and $\Phi: \operatorname{End}(X) \rightarrow \operatorname{End}(Y)$ the corresponding homomorphism, then $\varphi\left(\mathcal{V}\left(S, x_{0}\right)\right)=\mathcal{V}\left(\Phi(S), \varphi\left(x_{0}\right)\right)$.

Proof. Recall that $\varphi$ is bijective. We have

$$
\begin{aligned}
x \in \mathcal{V}\left(S, x_{0}\right) & \Longleftrightarrow f(x)=x_{0} \text { for all } f \in S \\
& \Longleftrightarrow \varphi(f(x))=\varphi\left(x_{0}\right) \text { for all } f \in S \\
& \Longleftrightarrow \Phi(f)(\varphi(x))=\varphi\left(x_{0}\right) \text { for all } f \in S \\
& \Longleftrightarrow \varphi(x) \in \mathcal{V}\left(\Phi(S), \varphi\left(x_{0}\right)\right) .
\end{aligned}
$$

Remark 4.2. In general, not every closed subset $A \subset X$ is of the form $\mathcal{V}\left(S, x_{0}\right)$. However, if $X$ is affine and contains a copy of the affine line, then we have an embed$\operatorname{ding} \mathcal{O}(X)=\operatorname{Mor}\left(X, \mathbb{A}^{1}\right) \subset \operatorname{End}(X)$, and one gets $\mathcal{V}_{X}(S)=\mathcal{V}(S, 0)$ for $S \subset \mathcal{O}(X)$ and $0 \in \mathbb{A}^{1} \subset X$ where $\mathcal{V}_{X}(S) \subset X$ is the zero set of $S$. Hence, in this case every closed subset $A \subset X$ is of the form $\mathcal{V}\left(S, x_{0}\right)$.

Lemma 4.3. Let $\varphi: X \rightarrow Y$ be a conjugating map where $X$ is affine. If $A \subset X$ is a closed subset isomorphic to $\mathbb{A}^{1}$, then $\varphi(A) \subset Y$ is closed and the induced map $\left.\varphi\right|_{A}: A \rightarrow \varphi(A)$ is conjugating.

Proof. By the previous remark and Lemma 4.1 the subset $B:=\varphi(A) \subset Y$ is closed. Moreover, the restriction $\mathcal{O}(X) \rightarrow \mathcal{O}(A)$ is surjective. This implies that $\operatorname{Mor}(X, A) \rightarrow$ $\operatorname{Mor}(A, A)=\operatorname{End}(A)$ is surjective, because $A \simeq \mathbb{A}^{1}$. If $\alpha \in \operatorname{End}(A)$ and $\tilde{\alpha}: X \rightarrow A$ a lift of $\alpha$, then $\Phi(\tilde{\alpha})=\varphi \circ \tilde{\alpha} \circ \varphi^{-1} \in \operatorname{End}(Y)$ maps $B$ into itself, and $\left.\Phi(\tilde{\alpha})\right|_{B}=$ $\left.\varphi\right|_{A} \circ \alpha \circ\left(\left.\varphi\right|_{A}\right)^{-1} \in \operatorname{End}(B)$.

\section{Algebraic fields}

The basic ingredient in the proof of Theorem 2.5 is the following result.

Proposition 5.1. Let $\varphi: \mathbb{A}^{1} \rightarrow Z$ be a conjugating map. Then there is an isomorphism $\psi: Z \stackrel{\sim}{\longrightarrow} \mathbb{A}^{1}$ such that the composition $\psi \circ \varphi: \mathbb{A}^{1} \rightarrow \mathbb{A}^{1}$ is a field automorphism.

Proof. The variety $Z$ inherits from $\mathbb{A}^{1}$ the structure of a commutative field isomorphic to $k$ such that the additions $\alpha_{z_{0}}: z \mapsto z+z_{0}$, the multiplications $\mu_{z_{0}}: z \mapsto z_{0} z$ and the power maps $z \mapsto z^{n}, n \in \mathbb{N}$, are morphisms. It follows that $Z$ is smooth.

Let 0 resp. $1 \in Z$ be the identity elements for addition resp. multiplication, and put $Z^{*}:=Z \backslash\{0\}$.

(a) $Z$ is connected, hence irreducible. In fact, if $Z^{0}$ denotes the connected component of 0 , then any other component has the form $z_{0}+Z^{0}$. Since $0 \in Z^{0}$ and $0=z_{0} 0 \in z_{0} Z^{0}$ it follows that $z_{0} Z^{0}=Z^{0}$ for all $z_{0} \in Z^{*}$. Hence $Z=Z^{0}$, because $Z^{0}$ contains elements $\neq 0$. 
(b) Let $\ell$ be a prime different from char $k$, and denote by $\lambda: Z \rightarrow Z$ the power map $z \mapsto z^{\ell}$. Put $K:=\lambda^{*}(k(Z)) \subseteq k(Z)$ and denote by $K_{s} \subset k(Z)$ the separable closure of $K$ in $k(Z)$. Let $\tilde{Z}$ be the normal closure of $Z$ in $K_{s}$. Then we get the following commutative diagram where $\bar{\lambda}$ is finite and purely inseparable,

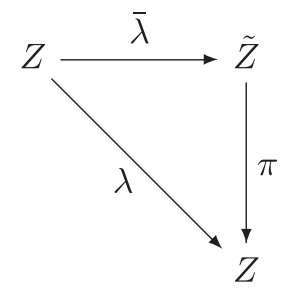

hence bijective, and $\pi$ is finite and separable. Moreover, the multiplications $\mu_{z}$ induce automorphisms $\bar{\mu}_{z}$ of $\tilde{Z}$ such that the following diagram commutes:

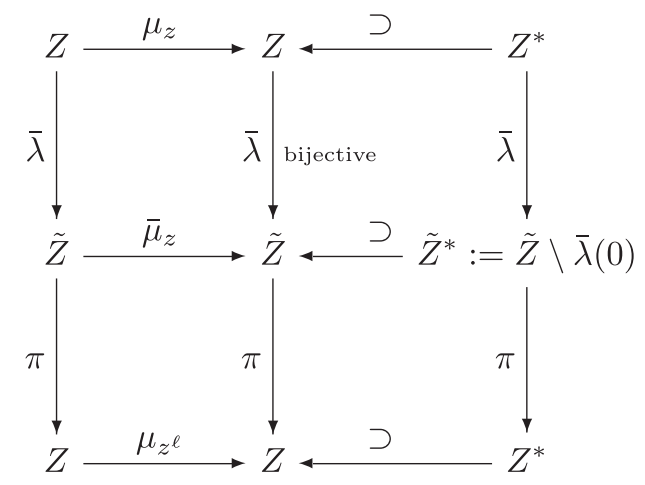

As a consequence, $\pi: \tilde{Z}^{*} \rightarrow Z^{*}$ is smooth, because $\pi$ is smooth on a non-empty open set $U \subset \tilde{Z}$ and therefore smooth in $\bar{\mu}_{z}(U)$ for all $z \in Z^{*}$.

(c) The map $\pi: \tilde{Z} \rightarrow Z$ is ramified in $\overline{0}:=\bar{\lambda}(0) \in \tilde{Z}$. In fact, consider the multiplication $\mu_{\zeta}$ where $\zeta$ is a primitive $\ell$ th root of unity. From above, we get the following commutative diagram:

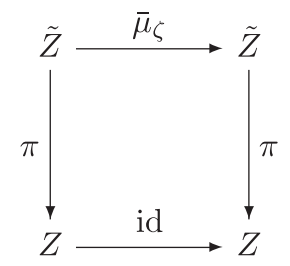

The differential of $\bar{\mu}_{\zeta}$ in $\overline{0}$ is a non-trivial automorphism of the tangent space $T_{\overline{0}} \tilde{Z}$, because the order of $\mu_{\zeta}$ is prime to $\operatorname{char}(k)$. Hence, $d \pi_{\overline{0}}: T_{\overline{0}} \tilde{Z} \rightarrow T_{0} Z$ cannot be an isomorphism.

(d) Now the "purity of the branch locus" implies that $\operatorname{dim} Z=1$ (cf. [AK71], [AK73]). Hence $Z$ is either an affine or a projective smooth curve. In both cases, $Z^{*}$ is a smooth affine algebraic curve whose automorphism group is infinite, and so $Z^{*} \simeq \mathbb{A}^{1} \backslash\{0\}$ or $Z^{*} \simeq \mathbb{A}^{1}$. In the second case, $\tilde{Z} \simeq \mathbb{P}^{1}$ which is impossible, because $\mathbb{P}^{1}$ has no automorphism of order $\ell$ with a single fixed point. Thus $Z \simeq \mathbb{A}^{1}$, and there is a unique isomorphism $\psi: Z \stackrel{\sim}{\rightarrow} \mathbb{A}^{1}$ with 
$\psi(0)=0$ and $\psi(1)=1$. It follows that $\rho:=\psi \circ \varphi: \mathbb{A}^{1} \rightarrow \mathbb{A}^{1}$ is a conjugating map with $\rho(0)=0$ and $\rho(1)=1$.

(e) It remains to see that $\rho$ is a field automorphism. The map $\rho \circ \mu_{z} \circ \rho^{-1}$ is an automorphism of $\mathbb{A}^{1}$ fixing 0 and sending 1 to $\rho(z)$, hence it is equal to $\mu_{\rho(z)}$. It follows that

$$
\rho\left(z_{1} z_{2}\right)=\rho\left(\mu_{z_{1}}\left(z_{2}\right)\right)=\left(\rho \circ \mu_{z_{1}} \circ \rho^{-1}\right)\left(\rho\left(z_{2}\right)\right)=\mu_{\rho\left(z_{1}\right)}\left(\rho\left(z_{2}\right)\right)=\rho\left(z_{1}\right) \rho\left(z_{2}\right) .
$$

Similarly, we see that $\rho \circ \alpha_{z} \circ \rho^{-1}$ is a fixed point free automorphism of $\mathbb{A}^{1}$ sending 0 to $\rho(z)$, hence is equal to $\alpha_{\rho(z)}$ which implies, as before, that $\rho\left(z_{1}+\right.$ $\left.z_{2}\right)=\rho\left(z_{1}\right)+\rho\left(z_{2}\right)$.

Remark 5.2. In case of $k=\mathbb{C}$ it suffices to consider only the additive structure on $Z$. A result of Palais' [Pal78] on separately polynomial maps then shows that $Z$ has the structure of an algebraic group. Using the Euclidean topology on $Z$ and the universal covering $\mathbb{C}^{d} \rightarrow Z$ one could conclude, as in the holomorphic setting (see [And11]), that $Z$ is isomorphic to $\mathbb{C}$.

\section{Proof of the main result}

For a variety $Y$ and a field automorphism $\sigma: k \rightarrow k$ we define $Y_{\sigma}:=\operatorname{Spec} k \times_{\operatorname{Spec} k} Y$ using the base change Spec $\sigma^{-1}:$ Spec $k \rightarrow$ Spec $k$. Thus, we have a canonical bijection $\pi_{\sigma}: Y_{\sigma} \rightarrow Y$ which sends closed sets into closed sets and induces an isomorphism $\operatorname{End}\left(Y_{\sigma}\right) \stackrel{\sim}{\longrightarrow} \operatorname{End}(Y)$, i.e., $\pi_{\sigma}$ is iso-conjugating. If $Y \subset k^{n}$ is defined by the polynomials $f_{1}, \ldots, f_{m}$, then $Y_{\sigma} \subset k^{n}$ is defined by the polynomials $f_{1}^{\sigma}, \ldots, f_{m}^{\sigma}$ where $\left(\sum_{i} c_{i} x^{i}\right)^{\sigma}:=\sum_{i} \sigma^{-1}\left(c_{i}\right) x^{i}$, and the map $\pi_{\sigma}: Y_{\sigma} \rightarrow Y$ is given by $\left(a_{1}, \ldots, a_{n}\right) \mapsto$ $\left(\sigma a_{1}, \ldots, \sigma a_{n}\right)$. In particular, $\left(\mathbb{A}^{n}\right)^{\sigma}=\mathbb{A}^{n}$ and $\pi_{\sigma}=\sigma$ in this case.

Proof of Theorem 2.5. We fix a closed embedding $\mathbb{A}^{1} \subseteq X$. Since $X$ is affine, the image of $\mathbb{A}^{1}$ is a zero set of the form $\mathcal{V}(S, 0)$ and so $Z:=\varphi\left(\mathbb{A}^{1}\right) \subset Y$ is closed and the induced map $\mathbb{A}^{1} \rightarrow Z$ is conjugating (Lemma 4.3). It follows from Proposition 5.1 that there is an isomorphism $\psi: Z \rightarrow \mathbb{A}^{1}$ such that the composition $\sigma:=\psi \circ \varphi: \mathbb{A}^{1} \rightarrow \mathbb{A}^{1}$ is a field automorphism. Composing $\varphi$ with $\pi_{\sigma}^{-1}: Y \rightarrow Y_{\sigma}$ and replacing $Y$ by $Y_{\sigma}$ we can assume that there is an embedding $\mathbb{A}^{1} \subseteq Y$ such that $\left.\varphi\right|_{\mathbb{A}^{1}}=\operatorname{id}_{\mathbb{A}^{1}}$ :

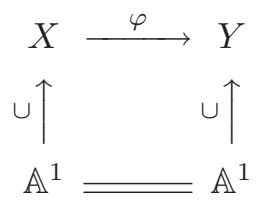

We thus obtain an embedding $\mathcal{O}(X)=\operatorname{Mor}\left(X, \mathbb{A}^{1}\right) \subset \operatorname{End}(X)$, and similarly for $Y$. Moreover, for $f \in \mathcal{O}(Y)$, we find

$$
\varphi^{*}(f)=f \circ \varphi=\varphi^{-1} \circ f \circ \varphi \in \operatorname{Mor}\left(X, \mathbb{A}^{1}\right)=\mathcal{O}(X) .
$$

The same holds for $\left(\varphi^{-1}\right)^{*}$ which shows that $\varphi^{*}: \mathcal{O}(Y) \rightarrow \mathcal{O}(X)$ is an isomorphism. Since $X$ is affine, it follows that $\psi:=\varphi^{-1}: Y \rightarrow X$ is a morphism inducing an isomorphism $\psi^{*}: \mathcal{O}(X) \stackrel{\sim}{\longrightarrow} \mathcal{O}(Y)$. Now the claim follows from Lemma 6.1 below. 
Lemma 6.1. Let $Y$ be a variety. Assume that $\mathcal{O}(Y)$ is finitely generated and that the canonical morphism $\psi_{Y}: Y \rightarrow \operatorname{Spec} \mathcal{O}(Y)$ is bijective. Then $Y$ is affine, and $\psi_{Y}$ is an isomorphism.

Proof. Zariski's Main Theorem in Grothendieck's form ([Gro67, Théorème 8.12.6], cf. [Mum99, Chapter III, Section 9]) implies that $\psi_{Y}$ admits a factorization $\psi_{Y}=\tau \circ \eta$,

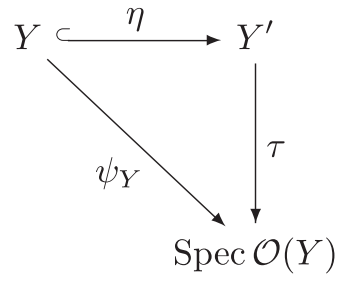

where $\eta$ is an open immersion and $\tau$ a finite morphism. From this we get inclusions $\mathcal{O}(Y) \subseteq \mathcal{O}\left(Y^{\prime}\right) \subseteq \mathcal{O}(Y)$, and thus $\tau$ is an isomorphism. It follows that $\psi_{Y}$ is a bijective open immersion, hence an isomorphism.

\section{Acknowledgments}

The authors would like to thank the referee for suggesting the construction of endofree varieties in Section 3, and Michel Brion for his help in this matter. The second author was partially supported by SNF (Schweizerischer Nationalfonds).

\section{References}

[AK71] Allen Altman and S.L. Kleiman, On the purity of the branch locus, Compositio Math. 23 (1971), 461-465.

[AK73] Correction to: "On the purity of the branch locus" Compositio Math. 23 (1971), 461-465; Composit. Math. 26 (1973), 175-180.

[And11] R.B. Andrist, Stein spaces characterized by their endomorphisms, Trans. Amer. Math. Soc. 363(5) (2011), 2341-2355.

[BM03] G.T. Buzzard and S. Merenkov, Maps conjugating holomorphic maps in $\mathbb{C}^{n}$. Indiana Univ. Math. J., 52(5) (2003), 1135-1146.

[Gro67] A. Grothendieck, Éléments de géométrie algébrique. IV. Étude locale des schémas et des morphismes de schémas (Troisième Partie), Inst. Hautes Études Sci. Publ. Math. (28) (1966).

[Har06] F. Hartogs, Zur Theorie der analytischen Funktionen mehrerer unabhängiger Veränderlichen, insbesondere über die Darstellung derselben durch Reihen, welche nach Potenzen einer Veränderlichen fortschreiten, Math. Ann. 62 (1906), 1-88.

[Har77] R. Hartshorne, Algebraic geometry, Graduate Texts in Mathematics, No. 52, SpringerVerlag, New York, 1977.

[Hin92] A. Hinkkanen, Functions conjugating entire functions to entire functions and semigroups of analytic endomorphisms. Complex Variables Theory Appl. 18 (1992), 149-154.

[Ere93] A. Eremenko, On the characterization of a Riemann surface by its semigroup of endomorphisms. Trans. AMS 338(1) (1993), 123-131.

[Mag75] K.D. Magill Jr., A survey of semigroups of continuous self-maps. Semigroup Forum 11 (1975/76), 189-282.

[ML96] L. Makar-Limanov, On the hypersurface $x+x^{2} y+z^{2}+t^{3}=0$ in $\mathbf{C}^{4}$ or a $\mathbf{C}^{3}$-like threefold which is not $\mathbf{C}^{3}$, Israel J. Math. 96 (1996), 419-429.

[Mer02] S. Merenkov, Equivalence of Domains with Isomorphic Semigroups of Endomorphisms. Proc. of AMS 130(6) (2002), 1743-1753.

[Mil08] J.S. Milne, Abelian Varieties (2.0), http://www.jmilne.org/math/CourseNotes/av.html, March 2008. 
[Mum99] D. Mumford, The red book of varieties and schemes, expanded ed., Lecture Notes in Mathematics, vol. 1358, Springer-Verlag, Berlin, 1999, Includes the Michigan lectures (1974) on curves and their Jacobians, With contributions by Enrico Arbarello.

[Pal78] R.S. Palais, Some analogues of Hartogs' theorem in an algebraic setting, Amer. J. Math. 100(2) (1978), 387-405.

Bergische Universität Wuppertal, Fachbereich Mathematik und Naturwissenschaften Gaussstr. 20, D-42119, Wuppertal

E-mail address: rafael.andrist@math.uni-wuppertal.de

Universität Basel, Fachbereich Mathematik und Informatik, Mathematisches Institut, Rheinsprung 21, CH-4051, BAsEL

E-mail address: hanspeter.kraft@unibas.ch 
